gave counting rates in a ratio $\frac{K \alpha(\mathrm{Ag})}{\mathrm{L} \alpha_{1}(\mathrm{Pt})}=4 \cdot 66$, and a lattice parameter $a=4.03 \AA$ (pure platinum, $a=3.9231 \AA$ ).

In the case of silver and a 0.002 molar solution of $\mathrm{Na}_{3} \mathrm{Au}\left(\mathrm{S}_{2} \mathrm{O}_{3}\right)_{2}$, the skin had the appearance of metallic silver and gave counting rates in the ratio $\frac{K \alpha(\mathrm{Ag})}{L \beta_{1}(\mathrm{Au})}=4 \cdot 71$. No measurements of lattice parameters were made because of the close similarity of the values for silver and gold (difference in $a=0.0076 \AA$ ).

Copper foil immersed overnight in a 0.002 molar solution of $\mathrm{Na}_{2} \mathrm{PdBr}_{4}$ and 4 molar sodium bromide gave a metallic skin with counting rates in the ratio $\frac{K \alpha(\mathrm{Cu})}{K \alpha(\mathrm{Pd})}=4 \cdot 07$, and lattice parameter $a=3.71 \AA$ (pure copper, $a=3.6150 \AA$; pure palladium, $a=3 \cdot 8898 \AA$ ).

From the lattice parameters it is found that the alloys contain more than 50 atom per cent of the less noble metal.

Attempts to obtain alloys of copper-silver failed, the skins invariably consisting of pure silver. This is not surprising, as the solubility of copper in silver is only 0.3 atom per cent at $100^{\circ} \mathrm{C}$ (ref. 6).

Research Laboratories,

H. Hirsch

Kodak, Ltd.,

Wealdstone,

Harrow, Middlesex.

Received January 5,1967 .

'Hund, F., and Trägner, E., Naturwissenschaften, 39, 63 (1952).

${ }^{2}$ Hund, F., and Mosthof, H., Naturwissenschaften, 39, 209 (1952).

'Hund, F., and Müller, J., Naturwissenschaften, 38, 303 (1951).

${ }^{4}$ Kulifay, s. M., J. Amer. Chem. Soc., 83, 4916 (1961).

${ }^{5}$ Holt, E. L., Nature, 203, 857 (1964).

Ageew, N., and Sachs, G., Z. Physik., 63, 293 (1930).

\section{Influence of Oxygen on Photoconduction in Organic Solutions}

We wish to give further results of our work on photoconduction in organic compounds at room temperature. We have already proposed $^{1}$ that photoconduction in solutions of triphenylamine in benzene and $n$-hexane requires the absorption of two quanta by the solute. Pilloff and Albrecht have recently shown a similar phenomenon in solutions of $N, N, N^{\prime}, N^{\prime}$-tetramethyl- $p$ phenylenediamine (TMPD) in 3-methylpentane ${ }^{2}$. Furthermore, our observations show that the magnitude of the photocurrent can be greatly reduced by changing the solvent from benzene to $n$-hexane. Wo have also described the influence of oxygen on the response of the pure solvent ${ }^{3}$.

Using the same experimental conditions, that is direct current measurements with a 'Teflon' and quartz cell operating with field strengths up to $20,000 \mathrm{~V} / \mathrm{cm}$, work has been carried out on solutions of triphenylamine saturated with dry oxygen. In benzene, the photocurrents were reduced to approximately 60 per cent of their usual values, while in $n$-hexane (where photocurrents are about two orders of magnitude less) there appeared to be no significant changes.

Analogous experiments have been carried out using dry nitrogen free from oxygen. In this case, with benzeno, the photocurrent values increased by approximately 10 per cent. Again, in $n$-hexane there were no significant changes.

Under all these conditions, it was found that the photocurrent was proportional to the light intensity and to the square root of the triphenylamine concentration, that is, that two photons were required for photoconduction, in agreement with the previous results and our interpretation of them.

The earlier work ${ }^{2}$ has also been extended to solutions of anthraceno in bonzene and $n$-hexane. Apart from an overall reduction in the observed photocurrents to about
5 per cent of the values obtained with triphenylamine, the results already stated have been reproduced. Oxygen and nitrogen saturation produced effects of a different magnitude from those given for triphenylamine, but the results are otherwise similar.

The main features of the photoconductivity of organic solutions are therefore similar when chemically different solutes are used; this has important implications in a more detailed explanation of the process.

As has been suggested elsewhere, for example by Brocklehurst et al. ${ }^{4}$, it is reasonable to consider the triplet state as a possible intermediate in a two-photon ionization process, especially in view of its long lifetime. In our work, flash photolysis confirmed our expectation that the oxygen-saturated solution showed no absorption by triplet state triphenylamine, whercas the solution saturated with nitrogen showed a strong triplet absorption. As already mentioned, the photocurrents were nevertheless only in the approximate ratio of $1: 2$. If the triplet state had been a rate determining intermediate species, we should have expected a far greater reduction of photoeurrent on saturation with oxygen.

A further argument against the participation of the triplet state is derived from our observation that, on saturating with nitrogen a solution of triphenylamine in equilibrium with air, the time taken for the triplet concentration after flashing to decay to half its initial value increased from $15 \mu \mathrm{sec}$ to $340 \mu \mathrm{sec}$. If absorption of a photon by the triplet state were needed for ionization, we might therefore expect a very large increase in photoconduction, which was not observed.

These results are also consistent with the observation that, in equally concentrated solutions of triphenylamine in benzene and $n$-hexane in equilibrium with air, the concentration of triplet state triphenylamine after flashing was approximately the same in both solvents, although the photocurrents differed by two orders of magnitude. Regarded in isolation, this change could be ascribed to a corresponding change of mobility or lifetime of the charge carriers in the two solvents. The possibility of attributing part of the change to this cause is not, of course, excluded.

It is interesting to compare these results with those of Johnson and Albrecht ${ }^{5}$, who studied the photoconduction of TMPD in rigid glasses at $77^{\circ} \mathrm{K}$. They, too, deduced at mechanism involving two photons and, among other interesting results, showed that saturation with oxygen reduced the photocurrent to roughly half its value in a helium-saturated rigid matrix. On this basis Johnson and Albrecht concluded that the triplet state of TMPD was not a rate determining step in the production of charge carriers.

On the evidence presented here it seems likely that the triplet state of the solute does not play an important part in photoconduction. The identification of the intermediate, and in particular its lifetime, is an important question. Johnson and Albrecht have suggestod that the solvent is involved, and certainly our results for $n$-hexane and benzene could be interpreted as supporting this proposal. There remain, however, many uncertainties which we hope to resolve by further experiment.

$$
\begin{aligned}
& \text { E. Pitts } \\
& \text { G. C. Terry } \\
& \text { F. W. Willets }
\end{aligned}
$$

Research Laboratories,

Kodak, Ltd.,

Wealdstone,

Harrow, Middlesex.

Received January $5,1967$.

${ }^{1}$ Pitts, E., Terry, G. C., and Willets, F. W., Trans. Faraday Soc., 62, 285̃1, $2858(1966)$

2 Pilloff, H. S., and Albrecht, A. C., Nature, 212, 499 (1986).

3 Pitts, E., Terry, G. C., and Willets, F. W., Nalure, 210, 295 (1966).

4 Brocklehurst, B., Gibbons, W. A., Lang, F. T., Porter, G., Savadatti, M. J., Trans. Faraday Soc., 62, 1793 (1966).

sohnson, G. E., and Albrecht, A. C., J. Chem. Phys., 44, 3162 (1966). 